\title{
Acknowledgement concerning two papers on rational dimension
}

\author{
by
}

\author{
S. D. Iliad is (Patras)
}

Concerning my paper: Rational spaces and the property of universality, Fund. Math. 131 (1988), 167-184, I would like to observe the following.

The main result of this paper is contained in the paper of G. Nöbeling, Über die rationale Dimension, Math. Ann. 109 (1934), 353-375.

Unfortunately, I had not come across this paper of G. Nöbeling in the literature. I have been informed quite recently about its existence by R. Engelking to whom I express my gratitude.

However, I would like to underline the following.

1. The method used in my paper is completely different from that of G. Nöbeling and it can be developed not only for $n$-dimensional rational spaces (see the paper Rational n-dimensional spaces and the property of universality (in Russian) by D. N. Georgiou and S. D. Iliadis, Fundamental and Applied Mathematics 4 (1998), no. 1, 49-74) but for some other classes of spaces as well.

2. Using my method one can prove some properties of universal spaces (for example, the property of finite intersections concerning simultaneous embeddings of compact rational spaces); I do not see how these could be proved by the method of G. Nöbeling.

Department of Mathematics

University of Patras

Patras, Greece

E-mail: iliadis@math.upatras.gr

Received 4 March 1998 